\title{
Le retour du père
}

The Father's Return (Lobi, Burkina Faso)

\section{Michèle Fiéloux}

\section{OpenEdition}

Journals

Édition électronique

URL : http://journals.openedition.org/span/1398

DOI : $10.4000 /$ span. 1398

ISSN : 2268-1558

\section{Éditeur}

École pratique des hautes études. Sciences humaines

\section{Édition imprimée}

Date de publication : 1 décembre 1994

Pagination : 159-184

ISSN : 0294-7080

\section{Référence électronique}

Michèle Fiéloux, «Le retour du père », Systèmes de pensée en Afrique noire [En ligne], 13|1994, mis en ligne le 17 février 2014, consulté le 19 avril 2019. URL : http://journals.openedition.org/span/1398 ; DOI : $10.4000 /$ span.1398 


\section{LE RETOUR DU PÈRE}

\section{par}

\section{Michèle Fiéloux}

Le rite lobi des secondes funérailles ou bobuùr ${ }^{1}$ est célébré pour les hommes et les femmes qui, au moment de leur décès, remplissent un ensemble de conditions dont la plus déterminante est l'accès au sixième grade initiatique ${ }^{2}$. On y parvient à un âge avancé, c'est-à-dire vers 55-60 ans, les femmes apres la ménopause, les hommes lorsqu'ils sont devenus des chefs de maison à part entière, responsables de leur unité de production, au niveau économique et religieux. On célebre un rite particulier, bobuùr khad, pour les doyens du lignage qui ont atteint ou dépassé le neuvième grade. La complexité des rites funéraires

${ }^{1}$ Nous avons systématiquement utilisé le lexique établi par Madeieine Père (1988). Elle donne pour bobuìr (p. 166) la composition suivante : bo "deviner", buìr "fonction de".

${ }^{2}$ L'initiation, $j \mathfrak{\jmath} r \dot{\jmath}$, est célébrée tous les sept ans. L'enfant se fait initier pour la première fois entre 7 et 10 ans. A l'initiation suivante, quand il a environ 14-17 ans, il devient l'éducateur (ou, en lobi, le "berger") des néophytes de la promotion qui suit la sienne. Il devra suivre six autres initiations pour accéder au statut d'aîné ( $k$ otin) qui lui donne droit au rite du bobviur.

Le deuil et ses rites III

Systèmes de pensée en Afrique noire, 13, 1994 
traduit la multiplicité des niveaux de différenciation sociale ${ }^{3}$. Entre le sixième et le neuvième grade, les variantes du bobuùr sont déterminées par le sexe, l'âge, le statut familial, la personnalité, les activités, les talents, la richesse du défunt. D'autres spécificités résultent de son appartenance à des unités territoriales précises et à différents groupes - patri et matriclans, confréries de chasseurs ou de devins, etc. Malgré cette grande diversité sociale et culturelle, on peut repérer trois dimensions essentielles dans ce rituel; nous nous attacherons plus particulièrement à la troisième d'entre elles, liée à la notion de culte des ancêtres.

1) Une dimension théorique qui permet de décrire le passage d'un monde à un autre. Passage particulièrement délicat et dangereux où progressivement le défunt perd la parcelle de vie qu'il détenait encore au moment de son enterrement pour devenir un mort achevé, prêt à entrer dans "le pays des morts", khididus. Parallèlement, les proches $\mathrm{du}$ défunt, en particulier les veufs qui sont traités pendant toute la période du veuvage comme les "jumeaux du mort"4, se détachent progressivement de lui; ils retrouvent une pleine vitalité, et la veuve, sa véritable capacité de procréation. La logique qui est ici à l'œuvre emprunte au processus de transformation du mil en bière. Les états successifs: graine germée, graine écrasée, bière de mil non fermentée

${ }^{3}$ Nous décrirons exclusivement les rites réservés aux hommes décédés alors qu'ils étaient entre le sixième et le neuvième grade initiatique. Les enquêtes ont été menées dans les cantons d'Iridiaka et de Boussera, dans le sud-ouest du Burkina Faso, principalement dans les villages de Vourbira, Gomboulora, Pleingua, Korogho, Tyorgbalandi, Sewcra et Boussera. Notre analyse est principalement fondée sur l'observation des secondes funérailles organisées en 1988 pour Binduté Da. En collaboration avec $\mathrm{J}$. Lombard, nous avons réalisé un film sur ce rite (Fiéloux et Lombard, 1990). Nous présentons les conditions du tournage ainsi qu'une description de ce rite dans Les Mémoires de Binduté Da (sous presse). Certains passages de cet article sont extraits de ce dernier ouvrage.

L'ouvrage de référence sur les rites funéraires (Goody, 1962) est consacréà une population culturellement proche des Lobi, les Dagara.

4 Cette notion de gémellité sc comprend par rapport aux liens unissant les doubles, celui du défunt et celui de la veuve. On considère qu'au bout de plusieurs années de vie conjugale, les doubles sont comme "ajustés" l'un à l'autre, que la femme ait eu ou non des enfants. Nous avons développé cet aspect dans la description du rite réservé à Binduté Da. 
et fermentée, résidus de la première et de la seconde cuisson, correspondent à autant d'étapes de la construction de l'ancêtre et dans un mouvement concomitant, à celles de la levée de deuil. L'ensemble de ces transformations constitue la cérémonie dans son unité de temps. Alors que la germination amorce la première étape de la levée de deuil, la consommation collective de la bière de mil du défunt, khíditaàn ${ }^{5}$, ultime moment de convivialité, signifie la séparation définitive des deux mondes, celui des vivants et celui des morts, et l'achevement du rituel.

2) Une dimension que nous appellerons celle de l'épopée, qui s'exprime par des mimes, des chants, des danses s'appuyant sur des images, des objets, des reliques, des évocations de la vie du défunt, pour libérer les imaginaires tout en les canalisant, et qui va permettre l'élaboration d'une représentation définitive du futur ancêtre. Ces évocations qui, pour la plupart, ont déjà été mises en scène au cours des premières funérailles (bii, de biir: pleurer) constituent un bilan, jamais dressé au hasard, qui établit de fait une véritable hiérarchie entre les morts et dévoilent les valeurs stéréotypées de la société lobi. Ces scénarios sont en partie réalisés par ceux là même qui pourront, d'une manière ou d'une autre, prendre la place laissée vacante, et poursuivre l'cuvre du défunt ou de la défunte. Les acteurs sont recrutés parmi ses propres descendants, ou dans le "groupe de ses semblables", membres des mêmes confréries, comme celle des guerriers honorifiques, encore appelés "hommes amers", kuunkhaa ${ }^{6}$, ou des mêmes communautés: chasseurs d'éléphants initiés au même culte, orpailleuses originaires du même village, devins partageant des savoirs communs, etc.

3) En troisième lieu, le rite du bobuùr peut apparaître comme un code qui permet de décrire le système d'organisation sociale et d'en assurer la reproduction. Il met en cuvre un double jeu de représentations. Le premier renvoie aux conceptions relatives à la personne, tibil,

${ }^{5}$ L'origine du mil est une donnée importante. Ici, la bière de mil du défunt, faite avec le mil provenant du grenier du défunt, ou acheté avec ses revenus propres, est utilisée à des moments très précis du rite.

${ }^{6}$ Voir P. Bonnafé et M. Fiéloux, 1984. 
dont l'un des constituants, le thuù, que nous traduirons tout au long du texte par le terme "double", survit apres la mort, dissocié du corps considéré alors comme une enveloppe vide. On suppose que cette dissociation s'est opérée avant la mort réelle, trois ou quatre ans avant selon le sexe du défunt ${ }^{7}$. Pendant la période intermédiaire entre les premières funérailles (ou rites d'enterrement) et le bobuùr, le double du défunt change d'apparence comme le cadavre lui-même. Il subit lui aussi les signes de l'altération, de la décomposition, et passe de l'image concrète d'un être humain, tibil, que les "clairvoyants" peuvent identifier par ses traits, sa démarche, son allure générale, à l'image abstraite, "épurée", d'un cadavre sec, détaché du monde des vivants et privé de toute capacité à s'immiscer dans ce "bas-monde" pour se venger. Plus le bobuùr approche et moins l'on se protège du défunt. Les rites de levée de deuil confirment cette séparation et écartent tous les dangers apportés par le mort qui se trouvait jusque là davantage du côté des vivants que du côté des morts. Le double, thuù, peut alors entreprendre le voyage qui fera de lui non plus l'image d'une personne, tibil, mais la représentation d'une puissance invisible, thil ${ }^{8}$. Voyage conduisant selon des itinéraires précis sur l'autre rive de la Volta Noire, d'où sont venus les premiers immigrants lobi dans la deuxième moitié du 18ème siècle.

Le second jeu de représentations engage un mouvement inverse qui assure le passage de cette puissance thil du monde des morts à celui des vivants par sa représentation ou sa "présentification" ${ }^{\text {9 }}$ dans un lieu de culte approprié. La construction d'un véritable ancêtre implique une longue gestation, comme le laisse supposer l'esquisse du futur lieu de culte, première image de l'ancêtre, commencée pendant le rite du bobuùr, mais qui ne sera achevée qu'au moment de "la construction définitive", plusieurs années après la fin du bobuùr.

${ }^{7}$ La différence des sexes est marquée par le chiffre 3 masculin et 4 féminin.

8 Il existe des thila (sing. thil) claniques, sous-claniques, lignagers et individuels. C'est à la fois l'autel concerné, la puissance et son support ancestral (Bonnafé \& Fiéloux, 1984: 83).

${ }^{9}$ Ce terme a été emprunté au travail de J. P. Vernant, Figures, idoles et masques, Paris, Julliard, 1990. 
Toute l'histoire post-mortem se résume dans le nom attribué au lieu de culte définitif, qui installe le défunt à sa juste place dans l'ordre généalogique, et l'investit d'une force supplémentaire comme "père", thire, pour ses descendants, réaffirmant ainsi la préminence du rôle paternel.

Toute personne est identifiée, dans sa parenté, par une triple relation (Père, 1988: 149): I'une à son propre matriclan, cadr, et à ses segmentations (sous-matriclan localisé, matrilignage), l'autre au matriclan de son père, thicaàr, à l'intérieur duquel se recrutent, selon la terminologie de parenté, les "pères classificatoires" ou les substituts du "père", et la troisième à son patriclan. Ce troisième groupe de référence, connu par le terme générique kuon, accorde une place prépondérante au "père" (et à ses ancêtres agnatiques) qui, selon les conceptions lobi, peut "donner naissance" ( kuon $\left.^{10}\right)$ en affiliant par un rite spécifique $^{11}$ tout enfant à son propre patrilignage. De même, la grande initiation $(j 3 r 3)$, étape indispensable pour l'acquisition de l'identité lobi, s'effectue selon des rites propres à chaque groupe patrilinéaire. Par ailleurs, "un médicament", thiikuon, provenant de l'arbre sacré, symbole du kuon et dont une branche, plantée devant la porte de la maison, sert d'emblème identitaire, thilkhad, concrétise le concept "biens du père", thitiz, utilisé pour définir les différents domaines d'intervention des ancêtres agnatiques. Par l'intermédiaire du "père", le kuon intervient, avec des rites propres, à toutes les étapes de la vie: naissance, initiation, acquisition de l'autonomie économique, construction de maison, éventuellement migration, et par l'intermédiaire d'un père "classificatoire", à l'enterrement, au départ vers l'au-delà et à la construction du lieu de culte du père ancestralisé, thire. On doit souligner enfin le rôle prépondérant joué dans les activités économiques par

${ }^{10}$ Kuon peut être traduit par engendrer, procréer, donner naissance. Le même terme est employé pour désigner le patrilignage (Père, 1988: 108).

${ }^{11}$ Le rite du nuokhati: "médicament amer de la bouche" (Père, 1988: 147). 
le kuon qui impose des règles très strictes concernant la production, la consommation et l'utilisation des revenus ${ }^{12}$.

Au cours du bobuùr sont mises en scène certaines des fonctions propres à ces groupes de parenté. Ainsi le groupe matrilinéaire d'ego, dans lequel se transmet la richesse (bétail, cauris, argent, volaille, produits récoltés) et les personnes (droit de garde des enfants, remariage des veuves sans compensation matrimoniale), constitue un groupe solidaire de défense armée. Ce principe est illustré, à la fin du rite, par le partage "des affaires du bobuùr" (bobuùr tiž): chaque proche parent utérin reçoit une fleche provenant de l'un des carquois du défunt ${ }^{13}$. A l'intérieur de ce même groupe, se recrutent un ou plusieurs pères adoptifs, qui prennent en charge les jeunes enfants d'ego, car la répartition des biens ne coïncide pas nécessairement avec celle des enfants ou des veuves.

Le rôle du matriclan du père, thicaàr, consiste à intervenir à travers l'un de ses membres, reconnu comme "père" du défunt, pour l'aider à franchir les différentes étapes de son voyage vers l'au-delà. Par exemple, il intervient pendant les premières funérailles, lors de l'interrogatoire sur les causes de la mort, puis "ouvre la tombe" en fournissant la poule et les cauris au fossoyeur, et pendant le bobuùr, "donne accès au pays des morts" en prononçant les dernières salutations.

Le groupe patrilinéaire, kuon, est omniprésent. Il se manifeste par de nombreux signes, souvent très discrets, comme le médicament, thiikuon, déposé sous la jarre installée face à la porte de la maison qui représente la personne pour laquelle on célèbre le bobuùr. Il commande également certains rites dont celui de la levée de deuil.

12 Les Lobi font une distinction fondamentale entre produits "amers" et produits "froids", variable selon les patriclans et qui concerne les produits de l'agriculture, de la chasse, de l'artisanat, etc. Voir Fiéloux, 1980, Père, 1988 et Bonnafé, 1993.

13 L'exclusion d'un matriclan, caàr, prononcé par exemple en cas d'homicide d'un utérin, s'appliquera dans ces circonstances, puisque l'exclu ne peut aucunement se mêler à ses parents utérins et recevoir la flèche, image emblématique du guerrier qu'il doit incarner pour prêter assistance (ou pour se substituer) à ses utérins, ses "semblables", biel. 
Enfin, comme nous le verrons, les espaces rituels choisis pour opérer la transformation du double thuù en puissance thil sont particulièrement investis par le patrilignage, puisque s'y trouvent toutes choses venant "du père": autel du père d'ego, grenier de mil, bétail, etc.

Les conceptions relatives à la construction d'un ancêtre et du lieu de culte correspondant s'expriment à travers les manipulations, le plus souvent interdépendantes, de deux types d'objet: une relique et un objet familier.

Du double à la puissance, du thuì au thil, du monde des vivants à celui des morts.

Au terme des premières funérailles (bii, "pleurs"), on établit une représentation du double du défunt à l'aide d'une relique appelée également bii, qui est composée d'objets associés à un certain état du mort (cadavre sur le point d'être enterré), dont un morceau de la nattelinceul et du brancard ayant servi lors de l'interrogatoire, et des cauris, offrandes viatiques, donnés pour le voyage du défunt dans l'au-delà. Cette relique, soigneusement gardée pendant la période intermédiaire, est si fortement associée à l'idée d'un mort "encore présent", et donc du côté des vivants, qu'on ne peut ni la toucher directement, ni abandonner la maison qui l'abrite. Tout est fait pour empêcher qu'elle serve d'objet magique pour nuire aux parents utérins du défunt, conformément aux pratiques de vengeance exercées entre matriclans, caàr. De plus, le vol de la relique, qui empêcherait la célébration du rite du bobuùr, équivaudrait à faire du défunt un errant, privant les siens de son influence bénéfique. Ce sont, en effet, les multiples manipulations de cette relique et la transformation de ses propriétés au cours du rituel funéraire, qui vont réaliser la séparation du mort d'avec les vivants. Elle va ainsi passer du "chaud", puù, au "froid", yii, termes utilisés dans d'autres contextes pour indiquer le passage du "sacré", khaàr, toujours potentiellement dangereux, au profane. A cette transformation correspond un changement de statut de la relique bii, qui tout d'abord "présentifie" le double thuù du défunt pour devenir à la 
fin des secondes funerailles un objet insignifiant, comparable à n'importe quel déchet ou objet abîmé, cassé, inutilisable dont on souhaite se débarrasser. Ainsi on rend présent le double du défunt dans sa propre maison en y déposant le bii, trois ou quatre jours après l'enterrement, ce qui correspond au début de la prise de deuil. Inversement, on rend manifeste son absence, à la fin du bobuùr, en transportant la relique hors de la maison, de telle façon qu'elle représente "un homme sortant de chez lui". On lui imprime un mouvement de marche, en la soulevant et en la reposant à terre jusqu'au chemin conduisant au "pays des morts". Tant que le double est encore parmi les vivants, on déplace l'objet-relique, mais on ne le bouge plus quand le double a commencé son voyage vers l'au-delà.

De la Puissance à l'Ancêtre-Père, du thil au thírc, du monde des morts à celui des vivants.

Le passage de la puissance thil à l'ancêtre père thíre se fait par la manipulation d'un objet familier rappelant que le défunt était un homme âgé, ayant normalement des descendants de la seconde et même de la troisième génération. On utilise sa canne de marche, gbuù, tout au long du rite du bobuùr pour mettre en scène la gestation de l'ancêtre et son futur retour comme puissance-père, nommément invoquée et individualisée, dans sa propre famille. Cette canne n'est pas assimilable aux objets emblématiques conservés après l'enterrement, comme les armes pour un homme ou certains ustensiles ménagers pour une femme, et distribuées, nous l'avons vu, à la fin du bobuùr, à titre de bobuùr tič, comme des insignes d'appartenance à tel groupe de parenté. L'objet gbuù relève d'un autre type de représentation. On pourrait le décrire comme une image abstraite, un simulacre de la présence future de l'ancêtre. Lors du bobuùr, on commence les opérations rituelles sur la canne, notamment en découpant le pommeau, dès que le statut du double thuù le situe davantage du côté des morts que de celui des vivants. Le double du défunt subit, à l'aube du second jour du rite, une véritable métamorphose, et donc un changement d'identité, qui le fait "ressembler" aux ancêtres. Le processus de la 
transformation du mil en bière et le code des couleurs constituent l'essentiel des outils symboliques utilisés pour cette transmutation. On dit ainsi que les proches ancêtres du défunt venus de l'au-delà à cette occasion rendent le double semblable à eux en lui donnant la couleur des vrais morts, rouge braise, visible la nuit, au lieu de blanc, couleur des "cadavres vivants", comme le sont notamment les veuves encore liées au défunt. La modification du goût de la bière de mil ayant servi à "laver" le défunt sera interprétée comme l'accomplissement de la métamorphose du double. Aussi, ce changement de goût, de doux à aigre, jugé nécessaire à la fermentation du liquide, est la condition de la poursuite du bobuùr. Dès lors, le gbuù va figurer un "père" qui "ressemble" par sa couleur à un ancêtre mais qui ne vit pas encore dans l'au-delà et n'est pas investi du pouvoir reconnu à l'ancêtre. Ajoutons que l'on ne sait pas quel genre d'ancêtre il deviendra. Cette incertitude est clairement exprimée dans le bobuùr, puisque les proches du défunt, avant le départ du double dans l'au-delà, vont le supplier de devenir un bon ancêtre, de prendre soin de sa maison et des siens.

Sous cette première forme, l'objet ghuù sera associé à la représentation du double du défunt, la relique bii, pour constituer un lieu de culte provisoire, appelé précisément "défunt", khidi, ou "pleurs", bii. Les proches, suivant un ordre précis - "père classificatoire", parents utérins, veuves, enfants - feront face à cet autel pour demander au défunt de devenir "un bon ancêtre". Puis les deux éléments seront definitivement dissociés. Le double thuù poursuivra son chemin vers l'au-delà, départ exprimé par le fait de jeter la relique bii sur la route des morts, pendant que l'objet-gbuù sera progressivement transformé en une représentation "achievée" du futur ancêtre, à l'image du défunt qui est passé de l'état de "mort-encore un peu vivant" à celui de "mort intégral". Le traitement de l'objet gbuù, qui commence le troisième jour du bobuùr et prend fin plusieurs années après le rite, reproduit les grandes scansions du cycle funéraire: premier rite funéraire, période intermédiaire, second rite funéraire avec construction du lieu de culte "père", thíre. Ainsi, des le départ du double thuù dans l'au-delà, on traite le gbuù comme le défunt le jour de son enterrement, en lui prodiguant des soins équivalents: toilette, représentations honorifiques de sa vie, salutations, inhumation. En revanche, on ne mime pas 
l'interrogatoire du mort, phase importante du premier rite funéraire, puisque l'ohjet figurant le père correspond à un mort déjà purifié, pour lequel il n'est plus nécessaire d'expliciter les causes de la mort. L'objet-canne passe donc de l'état de gbuù (défunt dont le double thuù est encore présent ici-bas, le bobuùr n'étant pas achevé) à celui de gbàl (défunt dont le thuù est parti dans l'au-delà et qui constitue dorénavant une puissance, thil). L'état du gbàl est relativement figé pendant toute la période intermédiaire (entre la fin du bobuùr et la construction du lieu de culte "père") qui correspond à la gestation de l'ancêtre. Ainsi on "enterre" symboliquement le gbàl dans la maison où sera construit le lieu de culte définitif, le moment de l'inhumation se faisant parallèlement à "la naissance" de la puissance, thil, dans l'au-delà, destination ultime du parcours du double thuù. La durée de la période intermédiaire (dix à vingt ans) renvoie peut-être au cycle initiatique: l'ancêtre franchirait dans l'au-delà le dernier grade de l'initiation pour atteindre sa totale plénitude. La fin du cycle est généralement indiquée par un rêve, mode de communication entre les deux mondes, fait par le fils aîné du défunt, son successeur désigné. Dans une dernière métamorphose, le défunt devient un ancêtre, l'objet-canne cesse d'être nommé gbuì ou terme dérive, gbàl, pour devenir un "père", thíre.

Etant donné la faible profondeur de la mémoire généalogique, le "père" disparaîtra comme ancêtre nommé de la mémoire de ses descendants au bout de trois ou quatre générations pour se fondre dans le groupe des ancêtres anonymes, autre étape de son histoire. Au bout de quatre à cinq générations, le double thuù, élément vital impérissable, participe à la conception d'un enfant par lequel il entreprend une nouvelle existence sociale.

Nous allons maintenant tenter de démonter les mécanismes par lesquelles ces conceptions relatives à la personne et à son devenir comme ancêtre sont mises en auvre dans différentes opérations rituelles relevées dans le rite du bobuùr et dans les rites ultérieurs de construction de l'autel-père. 


\section{La canne du défunt: $g$ buù}

Au cours du rite du bobuùr, la première étape consiste à déterminer le lieu où résidera l'objet-canne gbuù pendant la période comprise entre la fin du rite du bobuùr et la construction du lieu de culte dénommé "père". Tout commence donc le troisième jour du rite dès l'instant où le double thuù, ayant changé de couleur, est devenu "semblable" aux ancêtres. On procède, à ce moment-là, à une opération divinatoire conduite symboliquement par le défunt lui-même, puisqu'on dépose tout-à-côté du devin, la relique bii ("pleurs"). On attend du défunt qu'il indique sa propre maison, comme s'il prouvait ainsi son attachement aux siens et sa volonté de devenir un bon ancêtre. Il peut également cohabiter avec des parents agnatiques, y compris dans la "grande maison" de l'initiation ou cor kotin ${ }^{14}$, ce qui renvoie, dans les deux cas, au rôle joué par le patrilignage dans les différentes activités économiques. En revanche, il parait inconcevable qu'il "trouve sa place" dans son propre matriclan, compte tenu des fonctions assignées à ce groupe de parenté.

Dans un deuxième temps, un musicien, spécialiste du balafon des funérailles ${ }^{15}$, construit l'autel provisoire, "défunt" ou "pleurs", en plantant le pommeau de la canne dans une motte de terre élevée dans un coin sombre de la chambre de la première épouse, pièce qui sert de reliquaire depuis l'enterrement. Toute la distinction entre l'ébauche de l'autel et son achevement comme "lieu du culte du père" tient à la manière de formuler les invocations. On ne dialogue pas avec le défunt, khidi, comme on le fera plus tard avec son "père", thire. On accompagne la première intervention d'offrandes correspondant au statut, au sexe, à l'âge, des donateurs: cauris, volaille, bélier, etc., viatiques pour l'ultime voyage. Aucun animal n'est utilisé comme objet divinatoire. Au moment de l'égorgement, le donateur doit tenir la volaille fortement serrée entre ses mains pour empêcher qu'on puisse

14 "La grande maison" est la maison de l'ancêtre du patrilignage, ou plus souvent les ruines qui en restent. $M$. Père, 1988: 110.

15 Il existe plusieurs catégories de balafons. Voir D. Branger, 1993. 
lire dans ses mouvements le moindre signe. Le défunt n'a pas encore droit à la parole, dans ce contexte précis. Il ne transmet des messages au cours du bobuùr que par l'intermédiaire du devin dont la présence exclut celle de l'autel provisoire: le devin doit avoir quitté les lieux pour que le musicien commence sa tâche.

Ce premier dialogue est qualifié par le terme jéfy, utilisé pour ce seul rite, qui évoque l'idée de séparation, puisqu'il indique le geste d'enlever prestement quelque chose qui vient de se poser sur soi. Il ne peut être accompagné de pleurs, tout particulièrement de la part des veuves. De nouvelles relations avec le défunt devenu "sec" doivent être établies. Ce changement coïncide avec le début des opérations rituelles de levée de deuil ayant en commun l'idée "de rupture de liens": lavage abondant, rite de l'arc brisé, etc. L'offrande du bélier faite, au nom des veuves, sur l'autel provisoire, khídi, est sous-tendue par la même idée de séparation. Elle ne doit laisser, comme trace matérielle, qu'un bout d'oreille et non du sang. L'animal est tué et dépecé sur la partie de la terrasse réservée au chef de maison et donc au défunt. La consommation d'une part de viande est obligatoire pour toute personne ayant été mise en contact, d'une manière ou d'une autre, avec le défunt ou ses représentations, les objets-reliques. Elle permet aux veuves d'être déliées de leur alliance avec le patriclan de leur mari et marque la levée de deuil. De même, elle purifie les principaux acteurs des deux rites funéraires: fossoyeur, joueur de balafon, responsable du rite de levée de deuil. A un autre niveau, le partage des nourritures, dont la viande, entre les parents utérins, les enfants et les alliés claniques, préfigure le mode de transmission et de dispersion des biens du défunt, et en ce sens traduit l'accomplissement du deuil. Enfin, "la consommation d'une partie de la viande" par le défunt lui-même permet de parachever la mise a distance du défunt. On pourrait dire "que le défunt mange" lorsque les rites de levée de deuil sont entièrement accomplis, autorisant les veuves, purifiées, "dé-gemellisées", à reprendre le cours normal de la vie. Face à l'autel provisoire, khidi, on dispose les plats préparés par chacune des veuves. Ils sont composés de bouillie de mil à peine cuite et d'un bout d'intestin et de foie dans une sauce au sang. Le musicien nourrit le défunt comme on le ferait pour un thil, en prenant trois morceaux dans chacun des plats, 
dont le foie, organe vital, élément des nourritures sacrificielles. On signifie alors que le défunt a véritablement perdu son aspect de cadavre et qu'il est en train de se transformer en puissance thil. A ce moment, son double thuù est prêt à gagner l'au-delà. Déjà, au moment du repas, on devine des signes annonciateurs de la séparation définitive, les veuves murmurent en pleurant des messages à transmettre aux parents et amis réunis dans l'au-delà. L'enchaînement des séquences indique que le processus doit suivre son cours des qu'il est enclenché. Aussitôt le rite du repas au défunt terminé, la responsable de la cérémonie détruit l'autel provisoire, réunissant dans une vieille calebasse la terre mélangée aux plumes et au sang des animaux sacrifiés et la relique $b i i^{16}$, figure du double thuù, mais elle en retire ce qui représentera le défunt revenu chez lui comme ancêtre, la canne gbuù.

\section{La canne du défunt: $g b a ̀ l$}

On met en scène, selon un certain ordre, le départ du double dans l'au-delà et celui de la figure du père pour la maison désignée. Tout d'abord, on place le double du défunt sur la route conduisant au "pays des morts". Dès son départ, signalé par le cri de l'initiation propre au patriclan du mort, qui ouvre "la route des morts", on s'occupe du gbuù, comme s'il s'agissait du défunt le jour de ses premières funérailles, en suivant les différentes séquences déjà indiquées. Selon les mêmes techniques que celles employées pour faire du défunt un mort identifiable par les ancêtres, on lave la canne gbuù dans la bière de mil. Cependant, le mil ne provient pas du grenier du défunt comme dans le premier cas, mais a êté prélevé dans les paniers offerts par les veuves, les enfants et certains parents utérins. On prépare cette bière à côté de celle du défunt, khíditaàn, et non à proximité des foyers

16 Il arrive que l'on ne jette pas la relique bii sur la route, notamment pour les personnes qui avaient des ennemis connus de leur vivant, même si, après le rite du bobuìr, eette relique est normalement devenue inutilisable pour des pratiques magicoreligieuses. On peut rattacher ce fait à l'ensemble des précautions prises pour se défendre des ennemis potentiels depuis l'interdiction des conflits armés. 
réservés aux veuves et aux enfants, inscrivant de cette façon une frontière symbolique entre le monde des vivants et celui des morts. Ce feu particulier interdit tous les autres. Une seule cuisson suffit pour faire une bière non fermentée qui ne doit pas servir de boisson mais de détergent pour désincruster le gbuù de toutes traces de sang, de plumes et de terre. Une fois purifie, ou "mis propre" selon l'expression lobi, l'objet-gbuù sera entreposé dans un lieu intermédiaire entre l'intérieur et l'extérieur de la maison. Il ne peut être reposé à l'endroit qu'il occupait dans la chambre de la première épouse, et n'est pas assez transformé pour qu'on l'expose à l'extérieur, comme le cadavre l'avait été, adossé à côté de la porte, le premier jour des funérailles. Cependant, il se rapproche davantage de ce qu'il deviendra dans l'étape rituelle suivante. Aussi le met-on à proximité d'une voie d'accès à l'extérieur de la maison, près de l'escalier principal, c'est-à-dire sur l'espace masculin de la terrasse. A ce moment, le gbuù est posé sur le tabouret du défunt, recouvert d'une couche de feuilles de karité, plante aux vertus apaisantes utilisée en de nombreuses circonstances ${ }^{17}$.

Au cours de la phase suivante, on reconstruit une "image" du futur ancêtre en organisant une série de scènes où l'on mime ses principales activités, en faisant ressortir les traits les plus singuliers de sa personnalité. Le défunt gbuù assiste depuis la terrasse au spectacle de sa vie, comme il l'avait fait assis à l'ombre d'un arbre pendant le rite des premières funérailles. Remarquons que le spectacle commence après le départ du thuù dans l'au-delà et concerne donc les "vivants", famille, voisins immédiats, habitants d'une même région. Puis, on fait franchir au gbuù une étape de plus dans l'itinéraire du retour en le plaçant à l'extérieur de la maison, sur le seuil de la porte. A cet endroit très particulier de l'espace habité, le gbuù, image du défunt

17 Le karité, le plus souvent associé à de la cendre, est d'une part utilisé pour atténuer, ou même pour annihiler une action jugée destructrice ou incontrôlable: possession, conflit armé, querelle violente dans un lieu public comme le marché, etc. D'autre part, cette plante sert de protection contre l'esprit du défunt pendant toute la période du veuvage; on n'en fait plus usage dès le début de la levée de deuil. On retrouve le karité parmi les objets cultuels de l'arbitre clanique (moldaàr) dont le rôle est d'empêcher les dérèglements de l'ordre social. 
rendue dès lors publique, devient gbăl ${ }^{18}$. Ce changement de nom, procédure utilisée dans I'initiation pour qualifier une modification de statut ou d'état, est associé au double départ du défunt.

Les rites funéraires sont l'occasion d'exprimer la singularité de la personne. Des éléments subtils sont utilisés pour affiner l'image que donnent les scènes mimées. Ainsi faut-il que le găal soit déposé sur une peau de bouuf, comme l'avait été le gbuù sur le tabouret du défunt, situation analogue à celle d'une personne assise sur un siege (pierre, natte, etc). Le contact direct avec la terre renvoie à l'état de souillure mais peut être jugé nécessaire dans les périodes précédant certains rites de purification: homicide, "meurtre" de certains grands animaux, veuvage. On peut associer le gbàl posé à même la terre au dénuement, à la pauvreté, à l'infortune, à un destin peu glorieux. Pour suppléer a cette image dévalorisante, il arrive que l'on tue un boeuf, même s'il ne provient pas du troupeau du défunt, afin de témoigner que ce dernier tut, malgré son apparente pauvreté, quelqu'un de respectable. La peau d'un bouf immolé pendant les premières funérailles évoque un ensemble de valeurs liées au travail et de relations entretenues avec les ancêtres patrilignagers auxquels on attribue, nous l'avons vu, un rôle déterminant dans la réussite des activités économiques. De préférence, on représente le défunt comme agriculteur, en prenant la peau du "bouf de la houe", victime dont on entrave les pattes avec une houe au moment du sacrifice. On peut également l'honorer comme commerçant, forgeron, chasseur, devin, etc., en prélevant l'animal dans le stock correspondant, puisque toute activité économique doit se traduire, à un moment donné, par une accumulation spécifique de bœufs.

En présence de ce ghàl très personnalisé, chacun peut intervenir en associant l'évocation d'un souvenir à une oftrande de caurisviatique, comme pendant les premieres funérailles (une partie de ces cauris ayant servi à fabriquer la relique bii). Comme toute chose mise en contact avec la mort, les cauris changent de propriété, passant du froid yii, à l'amer, khaàr, du profane au sacré, et ne peuvent en tant que tels être utilisés impunément. Trois anciennes veuves sont chargées

18 Jusqu'à présent, le terme gbàl n'a pu être traduit par aucun informateur. 
de "les refroidir" en les plongeant dans le pot de bière de mil du défunt, khĩditaàn, récipient qui a déjà contenu la bière utilisée pour la purification du gbuù. La répartition des cauris est faite entre les trois responsables et la spécialiste du rite de levée de deuil.

On peut opposer cette séance publique d'adieux à la cérémonie familiale intime célébrée devant l'autel provisoire, khĩdi, bien que les relations de parenté y soient mises en scène de la même manière. L'ordre des premiers intervenants fait apparaître le rôle imputé au "pere" du défunt, qui dans les deux cas, doit "ouvrir la parole", une parole qui a toute son efficacité, puisqu'elle induit presque simultanément une progression dans la transformation du défunt. On considère que le véritable départ du double thuù coïncide avec les dernières salutations du "père" mettant "son fils sur la route". Ce "père" classificatoire, membre du matriclan du père du défunt, thicaàr, sera choisi parmi les plus proches parents ${ }^{19}$.

Les mouvements contraires: les voyages du double, thuù et de sa représentation, gbàl.

Ces deux voyages qui doivent aboutir à des destinations diamétralement opposées, le double rejoignant l'au-delà, et son image "père" restant ici-bas parmi les siens, dessinent des itinéraires qui renvoient à une représentation de l'espace de la maison, du territoire villageois, de la frontière entre culture et nature, des axes reliant les mondes des morts et des vivants.

Dans un premier temps, l'axe migratoire passe d'abord par l'espace "masculin" d'une maison ${ }^{20}$ puis par l'espace identifié comme khidihuo, route des ancêtres, situé en direction de la Volta Noire,

${ }^{19} \mathrm{M}$. Père (1988: 137) donne la liste des membres du matriclan du père (thicaàr) appelés à jouer un rôle de substitut de père.

${ }^{20}$ Nos informations sur l'organisation de l'espace sont principalement tirées des travaux de Antongini et Spini $(1981,1993)$. 
fleuve-frontière au-delà duquel on situe, de manière imprécise, le lieu d'origine des Lobi et le séjour des morts.

Dans un second temps (associé à celui de la parole paternelle), le thuù suit un itinéraire lié à l'histoire de ses ancêtres agnatiques. En effet, le "chemin des morts" correspond, nous l'avons vu, aux axes migratoires dessinés au 18ème siecle depuis les lieux historiques de la traversée de la Volta Noire. Ces migrations sont périodiquement remémorées, en principe tous les sept ans, grâce à l'initiation, qui amene néophytes et initiés à remonter le temps en parcourant en sens inverse les trajets bien définis suivis par leurs propres ancêtres. Il faut noter la complexe imbrication de ces trois niveaux, histoire clanique du peuplement, initiation, ancestralisation, qui font appel au même axe mort/vie, ancêtre/ descendant, que l'on doit parcourir pour acquérir aussi bien l'identité lobi grâce à l'initiation que le statut d'ancêtre grâce aux rites du bobuùr et de la construction de l'autel du père.

Des travaux récents (Père, 1988 et 1993) sur l'histoire du peuplement et des groupes de parenté font ressortir la grande diversité des patriclans et donc la multiplicité des trajets "initiatiques" et assimilés. Ces mouvements s'inscrivent dans la division du pays lobi en différentes unités territoriales: "village, réseau de villages, région" (Bonnafé, Fiéloux \& Kambou-Ferrand, 1982). Comme nous l'avions noté, "les habitants d'une même région (comprenant une moyenne de 12 villages) observent quel que soit leur clan des règles cérémonielles et des fêtes communes", dont le jour de clôture du rite du bobuùr. Ce jour doit correspondre à celui du plus ancien marché de la région, qu'il soit ou non encore en activité. Par exemple, le marché de Tyol, dans la région d'Iridiaka, fondé à la fin du 19ème siècle, ne s'anime plus actuellement qu'à l'occasion de l'initiation et, dans l'imaginaire, pour le départ des aînés dans l'au-delà. Le marché, lieu de rencontre entre ces unités sociales dispersées et séparées, est conçu comme étant à la charnière entre les deux mondes. Il est un passage obligé d'un changement de statut, qu'il s'agisse de la reconnaissance publique du néophyte devenu "grand", ou du double qui, dans l'itinéraire inverse de celui de l'initié, passe par ce point pour rejoindre le "pays des morts", et devenir une puissance, thil. 
Dans le même temps, le gbàl est l'objet d'un dernier rite funéraire, "l'inhumation". Un gendre, le mari de la fille aînée, est chargé de transporter le gbàl dans la maison désignée, généralement la propre maison du défunt. Cette mission est jugée périlleuse, puisque le gbàl représente à ce moment-là un mort "que l'on met dans une maison" et se trouve investi d'une force funeste. Pour écarter tout risque de contagion, on cherche à neutraliser l'objet en prenant comme première victime expiatoire le messager lui-même, à qui l'on tente de rendre l'objet en le lui lançant avec énergie, ou bien en le pourchassant pour le battre, lui faisant ainsi courir un danger de mort. Le gendre sacrifie ensuite une poule sur le gbàl jeté à terre dans le vestibule de la maison, rite qui évoque un sacrifice du même type effectué par le fossoyeur avant d'ouvrir la tombe. Normalement, l'objet-gbàl, réintroduit dans la maison du défunt, sera ensuite disposé par les gens de la famille à la place qu'il occupera plus tard comme ancêtre-père, tout-àcoté de l'autel dédié au propre père du défunt. Cette proximité physique du père et du fils est une représentation du patrilignage et des modes de résidence dans l'au-delà.

Le transfert du ghàl est une étape importante du rite puisqu'il signifie que tout s'est passé comme convenu, que le défunt est désormais à sa place, et qu'à leur tour les survivants peuvent exister socialement comme héritiers, pères adoptifs, veuves autorisées à se remarier, etc. On signale le départ du gbàl d'un coup de fusil autorisant le partage des "affaires du bobuùr" qui préfigure, nous l'avons vu, les modes de dispersion des biens du défunt et symbolise la solidarité nécessaire entre les membres du même groupe matrilinéaire. Signalons que le fils aîné reçoit à cette occasion une fleche, qui représente d'une certaine façon l'emprise du pere sur son fils. 


\section{La maison démolie et reconstruite.}

La maison du défunt est soumise au même schéma dynamique de "déconstruction" et de "reconstruction". Soudainement, avec l'annonce de la mort, la maison "tombe en ruine", $k e^{21}$, bien qu'on ne puisse dès ce moment l'abandonner. On considere qu'elle est dans un état ambivalent mort/vie, compte tenu des représentations liées à la personne et à ses reliques. Dans un deuxième temps, la maison va subir, du fait de l'héritier et non plus du défunt, une nouvelle "démolition". Provoquée par l'héritage des biens transmissibles en ligne utérine, elle est effectuée quelques mois après le bobuùr, le jour dénommé "prise des carquois", togba $a^{22}$. L'idée de "démolition" correspond au fait qu'il ne reste symboliquement plus "trace du défunt" dans la maison, que l'héritage des biens a pu coïncider avec l'adoption des enfants, le remariage des veuves, la migration des aînés, provoquant de fait l'éclatement plus ou moins important du groupe domestique. Cependant, la disparition totale et irréversible du chef de maison et de son œuvre n'est pas pensable. Devant la famille réunie, l'héritier prendra en même temps les objets personnels y compris les carquois (excepté celui réservé aux fils) et le bétail (boufs, moutons, chèvres), mais il ne peut faire place vide. Il doit procéder à un échange symbolique en remettant à celui des fils, généralement le cadet, qui est appelé à résider dans la maison paternelle, une génisse appelée "vache des enfants", bina, prélevée sur le troupeau hérité afin que le croît de cet animal puisse un jour servir à "construire" le lieu de culte "père", thíre. Dès lors, on n'est plus tenu de maintenir la maison dans l'état

${ }^{21}$ Le terme utilisé (koé) qualifie quelque chose de détérioré, d'abîmé, et au sens figuré, une personne atteinte de souillure parce qu'elle a transgressé certaines règles de conduite. L'idée de souillure étant liée à celle de contagion, toute personne dans cet état doit se tenir à l'écart (ce qui équivaut à une sanction).

22 Cette expression renvoie à l'usage de faire emporter un carquois par l'enfant ou la veuve qui va rejoindre l'héritier utérin (ou un membre du même sous-matriclan que le défunt). 
où l'avait laissée le père, et l'on peut la "reconstruire" et modifier son agencement intérieur (ce qui est souvent nécessaire quand les veuves remariées ont abandonné leur chambre).

Les agissements prétés au double du défunt manifestent cette volonté de laisser toujours "quelque chose" qui empêche l'anéantissement absolu. Un pere de famille est censé emporter dans l'au-delà tout ce dont il a besoin pour revivre comme chasseur, musicien, forgeron, etc. Il exercera ces activités dans ce monde pensé comme "pacifique", comme l'indique le fait de "désarmer" symboliquement le double juste avant son départ. Il retrouve ceux qui avaient disparu avant lui (parents, chien, bétail, animaux de la brousse tués par lui, etc.), mais il ne peut accumuler de nouveaux biens. De même, le mort ne peut se reproduire ou acquérir de nouvelles puissances, thila. Cependant, il n'emporte pas tout ce qui lui appartient. Un pere de famille doit laisser aux siens de quoi reconstituer les thila, dont il s'était lui-même serví pour garantir paix, entente et santé dans la maison. S'il peut emporter avec lui l'emblème du thil, désigné comme étant "l'œil" de la puissance, il ne peut l'anéantir totalement, sauf s'il n'a plus de descendants vivants. Il doit laisser une part vivante, sensible, permettant à ses descendants de lui redonner vie, un jour ou l'autre. Les procédures de renouvellement des thila, qui engagent tout un processus de purification, balayage, nettoyage, etc. commencent environ trois ans après le bobuùr.

\section{Le retour du père.}

Le retour du père comme ancêtre dans la maison prendra plus de temps que le réveil de certaines de ses puissances, thila. Pendant une première période qui varie entre dix et vingt ans, le père "reste au soleil", expression signifiant que le lieu où l'on communique avec lui se situe à l'extérieur de la maison, confondu avec le khídihus ou route des ancêtres, terme qui ne renvoie pas à un lieu précis, mais à un 
champ de relations entre les deux mondes. La position du corps face à l'est, à la Volta Noire, ouvre la communication avec l'au-delà. Pendant cette période, l'objet-gbàl n'est pas utilisable comme médiateur et garde la qualité d'objet insignifiant. Lorsque l'aîné des fils fait le rêve attendu, il transmet le message: "il est temps de me mettre à l'ombre". Ce rêve, dont l'interprétation est confirmée par un devin, a un caractère contraignant. On ne peut refuser l'hospitalité au père qui réapparait dans la plénitude de son statut d'ancêtre. Aussi on décide d'accomplir le rite nommé de manière métaphorique: kuthíre, litt. "construire père", c'est-à-dire l'autel qui lui est dédié.

Les rites de construction de l'autel sont spécifiques à chaque patriclan, mais ont en commun le sacrifice, le plus souvent, d'un taureau, symbole masculin, croît de l'animal fourni au moment de l'héritage, et la présence d'un "père classificatoire" qui reçoit la part de l'animal réservée au père de son vivant. Ces rites prolongent ceux qui ont été réalisés pendant le bobuùr. Le jour fixé doit correspondre à celui où se tient le plus ancien marché de la région; le retour du père se fait suivant l'axe qui relie les deux mondes. L'autel thír est le résultat de la dernière manipulation du même objet: la canne du défunt - ou son équivalent ${ }^{23}$ - est progressivement passé du statut de gbuù à celui de $g b a ̀ l$, puis enfin à celui de thíre. On distribue aux fils des fragments de l'autel, afin qu'ils puissent établir chez eux un lieu de culte, bien qu'ils ne soient pas obligés de le faire. Dans tous les cas, ils peuvent continuer à dialoguer avec leur père, non plus en faisant face au "pays des morts" mais en se tournant dans la direction de l'autel principal construit dans l'ancienne maison du père (ou celle du fils aîné). On peut supposer que cette nouvelle orientation traduit l'individualisation de l'ancêtre, qui reste un parmi d'autres, mais qui est également celui qu'on invoque nommément dans une place assignée, lieu qui apparaît stratégique à l'intérieur de la maison. De cette

\footnotetext{
23 Une branche d'arbre peut être utilisée en lieu et place de la canne. On recherche particulièrement le bambou, avec lequel on fabrique par ailleurs la corde de l'arc.
} 
façon est fixée la mémoire généalogique, puisque le défunt occupe la place qui lui revient comme membre à part entière d'un patrilignage. On l'installe à côté de son propre père, en respectant l'ordre des générations: on doit toujours "bâtir" le grand-père paternel avant le père. Par ailleurs, on assigne aux "pères" la place qui leur revient dans le vestibule, gbàlanwo, en mettant l'autel dans un coin sombre de la piece, quelquefois en face du grenier. Cette partie de la maison, nous l'avons vu, est particulièrement investie par le patrilignage. Tout ce qui appartient à ses domaines d'intervention y est regroupé: grenier (contenant notamment le mil réservé aux rites patrilignagers), meule dormante, jarres pour la fermentation de la bière de mil, instruments agricoles, bétail acquis avec les revenus de l'agriculture. De même, le vestibule, en tant qu'espace sacralisé, est l'un des éléments significatifs des rites qui s'y déroulent. C'est là que, rituellement, se font et se defont les liens qui attachent une personne à un groupe patrilignager. Les enfants y viennent au monde. Lors de la levée de deuil, la veuve ira s'adosser au grenier pour subir le rite par lequel on va la délier de son mari et donc du patriclan de celui-ci. On y résout également les différends qui résultent de la distribution du mil entre co-épouses. On y traite les transgressions graves d'interdits liés au mil, comme l'adultère. Enfin, on y accomplit différents rites agraires, dont le thitad̀n, "bière de mil du père", et la thina, "bœuf du père", termes qui traduisent bien l'importance du principe agnatique. On n'en donnera pas ici une description, mais on peut souligner leur caractere contraignant: chaque année au moment des nouvelles récoltes, la vie de la maisonnée est comme arrêtée. On doit fabriquer la bière thitaàn avec les grains de mil de la précédente récolte, restés au fond du compartiment réservé aux rites patrilignagers, ou conservés précieusement dans une poterie sur la terrasse. Pendant tout le temps de la préparation, le chef de maison et sa première épouse, qui l'assiste, ne peuvent sortir de la maison, même s'ils apprennent ces jours-là, cas extrême, qu'un villageois vient de mourir. La célébration de ce rite ouvre un nouveau 
cycle, si bien qu'une famille ne peut surseoir à son projet de migration au-delà de cette date. Les migrants partent dès l'offrande faite au père, transportant avec eux l'emblème qui doit accompagner un homme tout au long de sa vie, le thíre, la figure du Père.

Michèle Fiéloux

C.N.R.S. - URA 94 


\begin{tabular}{|c|c|c|c|}
\hline & Temps & Séquences rituelles & Lieu \\
\hline 1 & $\begin{array}{l}\text { Fin des premières } \\
\text { funérailles } \\
\text { (bii de biir: pleurer) }\end{array}$ & $\begin{array}{l}\text { Constitution des objets-reliques } \\
\text { dont la relique-pleurs (bii), } \\
\text { représentation du double, } \\
\text { (thuii) du défunt, les objets } \\
\text { emblématiques, la canne } \\
\text { (gbuii) }\end{array}$ & $\begin{array}{l}\text { * Mur extérieur de la } \\
\text { maison (= emplace- } \\
\text { ment du cadavre) } \\
\text { * Pièce-reliquaire } \\
\text { (chambre de la première } \\
\text { épouse, ou vestibule) }\end{array}$ \\
\hline 2 & $\begin{array}{l}\text { Période intermédiaire } \\
\text { entre les premières et les } \\
\text { secondes funérailles }\end{array}$ & $\begin{array}{l}\text { Les réliques sont gardées à } \\
\text { l'intérieur de la maison qui ne } \\
\text { peut être abandonnée }\end{array}$ & Pièce-reliquaire \\
\hline 3 & $\begin{array}{l}\text { Secondes funérailles ou } \\
\text { bobusùr }\end{array}$ & & 1 \\
\hline 3.1 & Germination du mil & $\begin{array}{l}\text { * Début de la levée de deuil } \\
\text { * Cohabitation des monts venus } \\
\text { de l'au-delà el des membres de } \\
\text { la famille du défunt }\end{array}$ & Maison du défunt \\
\hline 3.2 & $\begin{array}{l}\text { Préparation de la bière de } \\
\text { mil. Fin de la première } \\
\text { cuisson }\end{array}$ & $\begin{array}{l}\text { * Première étape du processus } \\
\text { d'ancestralisation. Changement } \\
\text { de couleur du double (thuit) du } \\
\text { défunt }\end{array}$ & $\begin{array}{l}\text { * Lisière de l'espace } \\
\text { habité et cultivé } \\
\qquad \mid\end{array}$ \\
\hline \multirow[t]{3}{*}{3.3} & $\begin{array}{l}\text { La bière fermentée est } \\
\text { bonne à boire } \\
\text { (3è jour du rite) }\end{array}$ & $\begin{array}{l}\text { * Consultation divinatoire. } \\
\text { Déplacement de la relique- } \\
\text { pleurs (bii) près du devin. } \\
\text { Participation du double du } \\
\text { défunt. } \\
\text { * Départ du devin. Construc- } \\
\text { tion de l'autel-défunt (khidi) } \\
\text { avec la relique-pleurs (bii) et } \\
\text { la canne gbuñ. Dernier dialo- } \\
\text { gue avec le double du défunt. }\end{array}$ & $\begin{array}{l}\text { * Vestibule ou espace } \\
\text { masculin de la maison } \\
\text { du défunt } \\
\text { * Pièce-reliquaire }\end{array}$ \\
\hline & Fin d'après-midi du $3 \grave{\text { jour }}$ & $\begin{array}{l}\text { * Dernier repas donné au } \\
\text { double du défunt }\end{array}$ & $\begin{array}{l}\text { * Autel-défunt (khidî). } \\
\text { Pièce-reliquaire }\end{array}$ \\
\hline & Nuit du 3è jour & $\begin{array}{l}\text { * Destruction de l'autel-défunt } \\
\text { et départ du double sur la route } \\
\text { des ancêtres. Cri de l'initiation }\end{array}$ & $\begin{array}{l}\text { * Pièce-reliquaire puis } \\
\text { sortie de la relique- } \\
\text { pleurs (bii) à l'extérieur } \\
\text { de la maison }\end{array}$ \\
\hline
\end{tabular}




\begin{tabular}{|c|c|c|c|}
\hline & Nuit du 3غ jour & $\begin{array}{l}\text { * Traitement de l"objet-relique } \\
\text { (toilette de la canne gbuì). }\end{array}$ & $\begin{array}{l}\text { * Extérieur de la mai- } \\
\text { son. Limite de l'espace } \\
\text { habité et cultivé } \\
\text { * La canne (gbuù) puri- } \\
\text { fiée est placée sur la } \\
\text { partie masculine de la } \\
\text { terrasse }\end{array}$ \\
\hline 4 & 4è jour du rite & $\begin{array}{l}\text { * Scènes mimées de la vie du } \\
\text { défunt } \\
\text { * Dernières salutations publi- } \\
\text { ques. Changement d'identité } \\
\text { du défunt avec l'invocation du } \\
\text { "père". Départ définitif du } \\
\text { double. La canne (gbutu) } \\
\text { change d'appellation et porte } \\
\text { désormais le nom de gbàl. } \\
\text { " Le gendre du défunt déplace } \\
\text { le gbăl, el "l'enterre" en un } \\
\text { lieu désigné par divination } \\
\text { * Partage des affaires du } \\
\text { bobuìr et consommation col- } \\
\text { lective de la bière de mil. }\end{array}$ & $\begin{array}{l}\text { * La canne est toujours } \\
\text { sur la terrasse. } \\
\text { * La canne (gbuì) est } \\
\text { retirée de la terrasse } \\
\text { pour être placée à l'ex- } \\
\text { lérieur de la maison, sur } \\
\text { la seuil de l'entrée. } \\
\text { * Généralement, le gbàl } \\
\text { est entreposé dans le } \\
\text { vestibule de la maison } \\
\text { du défunt. Autel du } \\
\text { patrilignage. } \\
\text { * Maison et alentours }\end{array}$ \\
\hline 5 & $\begin{array}{l}x \text { mois après la fin du } \\
\text { bobuùr }\end{array}$ & $\begin{array}{l}\text { "Liquidation de l"héritage du } \\
\text { défunt, ou "prise du carquois" } \\
\text { (ıugbà). Démolition symboli- } \\
\text { que de la maison. Remise par } \\
\text { l"héritier d'une génisse à un } \\
\text { fils du défunt. }\end{array}$ & * Maison du défunt \\
\hline 6 & $\begin{array}{l}x \text { années après la fin du } \\
\text { bobuùr }\end{array}$ & $\begin{array}{l}\text { * Construction de l'autel du } \\
\text { père (kuthirè). Le double thuà } \\
\text { est devenu une puissance (thíl) } \\
\text { et le gbuù transformé en gbàl } \\
\text { puis en thire représente le } \\
\text { défunt qui a accédé au statut } \\
\text { d'ancêtre. }\end{array}$ & $\begin{array}{l}\text { * Autel du patrilignage. } \\
\text { Maison du défunt ou du } \\
\text { fils aîné. }\end{array}$ \\
\hline
\end{tabular}




\section{Références bibliographiques}

Antongini G. \& Spini T.

1981 Il cammino degli antenati. I Lobi dell'Alto Volta, Laterza, Bari, 239 p.

1993 "La maison lobi: le relevé, méthode et résultats", in Images d'Afrique et Sciences sociales, M. Fiéloux, J. Lombard et J. M. Kambou-Ferrand éds, Karthala, 142-157.

Bonnafé P., Fiéloux M. \& Kambou-Ferrand J. M.

1982 "Un vent de folie? Le conflit armé dans une population sans Etat: les Lobi de Haute-Volta", in Guerres de lignages et Guerres d'Etats en Afrique, J. Bazin \& E. Terray éds, Paris, Ed. des Archives contemporaines, 73-141.

Bonnafé P. \& Fiéloux M.

1984 "Le dédain de la mort et la force du cadavre. Souillure et purification d'un meurtrier lobi", Etudes Rurales, 95-96: 63-87.

Branger D.

1993 "Le xylophone à résonateurs multiples des Lobi", in Images d'Afrique et Sciences Sociales, M. Fiéloux, J. Lombard \& J. M. Kambou-Ferrand éds, Karthala, 460-469.

Fiéloux, M. \& Lombard, J.

1990 Les Mémoires de Binduté Da, film documentaire $16 \mathrm{~mm}$ couleur, 52'. Producteurs: la Sept/ORSTOM et ATRIA films.

Les Mémoires de Binduté Da, Paris, EHESS (Collection Anthropologie visuelle) (sous presse)

Goody, J.

1962 Death, Property and The Ancestors: A Study of The Mortuary Customs of The LoDagaa of West Africa, Standford, California.

Père, M.

1988 Les Lobi, tradition et changement, Laval, Siloé, 2 vol, 992 p.

1993 "Chronique des villages de la province du Poni en contribution à l'histoire du peuplement au Burkina Faso", in Images d'Afrique et sciences Sociales, M. Fiéloux, J. Lombard \& J. M. KambouFerrand éds., Karthala, 57-73. 